\title{
Peptic ulcers accompanied with gastrointestinal bleeding, pylorus obstruction and cholangitis secondary to choledochoduodenal fistula: A case report
}

\author{
BIN XI ${ }^{1,2}$, JUN-JUN JIA ${ }^{1,2}$, BING-YI LIN ${ }^{1,2}$, LEI GENG ${ }^{1,2}$ and SHU-SEN ZHENG ${ }^{1,2}$ \\ ${ }^{1}$ Key Laboratory of Combined Multi-Organ Transplantation, Ministry of Public Health, \\ Key Laboratory of Organ Transplantation; ${ }^{2}$ Division of Hepatobiliary and Pancreatic Surgery, \\ Department of Surgery, The First Affiliated Hospital of Zhejiang University, Hangzhou, \\ Zhejiang 310003, P.R. China
}

Received November 22, 2014; Accepted September 14, 2015

DOI: $10.3892 / \mathrm{ol} .2015 .3908$

\begin{abstract}
Peptic ulcers are an extremely common condition, usually occurring in the stomach and proximal duodenum. However, cases of peptic ulcers accompanied with multiple complications are extremely rare and hard to treat. The present case reinforces the requirement for the early recognition and correct treatment of peptic ulcers accompanied with multiple complications. A 67-year-old man presented with recurrent abdominal pain, fever and melena. The laboratory results showed anemia (hemoglobin $62 \mathrm{~g} / \mathrm{l}$ ) and hypoproteinemia (23 g/l). Abdominal imaging examinations revealed stones in the gallbladder and right liver, with air in the dilated intrahepatic and extrahepatic bile ducts. Endoscopic retrograde cholangiopancreatography failed due to a deformed pylorus. The patient was finally diagnosed with peptic ulcers accompanied with gastrointestinal (GI) bleeding, pylorus obstruction and cholangitis secondary to a choledochoduodenal fistula during an emergency pancreatoduodenectomy, which was performed due to a massive hemorrhage of the GI tract. The patient recovered well after the surgery.
\end{abstract}

\section{Introduction}

Although there is a general declining trend in the incidence of peptic ulcers, the disease remains common. Approximately 500,000 people develop peptic ulcers per year in the USA (1). They are mainly caused by infection of Helicobacter pylori, gastric acid, nonsteroidal anti-inflammatory drugs (NSAIDs)

Correspondence to: Professor Shu-Sen Zheng, Division of Hepatobiliary and Pancreatic Surgery, Department of Surgery, First Affiliated Hospital of Zhejiang University, 79 Qingchun Road, Hangzhou, Zhejiang 310003, P.R. China

E-mail: zjdxzss@163.com

Key words: gastric ulcer, duodenal ulcer, gastrointestinal bleeding, pyloric obstruction, choledochoduodenal fistula, cholangitis and protective factors (2). Peptic ulcers are diagnosed by endoscopy and can be treated with proton pump inhibitors, $H$. pylori therapy (according to the results of $H$.pylori testing), endoscopy or surgery according to the severity of the disease. At the same time, non-variceal upper gastrointestinal (GI) bleeding and other accompanying complications of peptic ulcers remain a major prevalent and clinically significant condition worldwide $(3,4)$. If affected patients neglect the warning symptoms (e.g. epigastric discomfort, abdominal pain associated with eating, pain that causes awakening at night, weight loss, loss of appetite and melena) and receive no effective treatment for the peptic ulcer, then one or more complications will develop. These complications will make the disease much more complicated, and more difficult to diagnose and treat. The present study reports the case of such a patient who was diagnosed with peptic ulcers accompanied with upper GI bleeding, pylorus obstruction and choledochoduodenal fistula (CDF), and was successfully treated.

\section{Case report}

On December 12, 2010, a 67-year-old man was admitted to The First Affiliated Hospital of Zhejiang University (Hangzhou, Zhejiang, China) due to recurrent abdominal pain, fever and melena. The patient had a 1-year history of recurrent upper abdominal pain without any treatment, and no history of non-steroidal anti-inflammatory drugs. At 3 months prior to the admission, besides the abdominal pain, the patient experienced a fever, which was controlled by antibiotic treatment, without any abdominal distention or vomiting. At 1 month prior to admission, the patient was admitted into a local hospital due to persistent melena. However, the attempt to find the etiology and relieve the symptoms was unsuccessful. The patient was subsequently admitted to The First Affiliated Hospital of Zhejiang University for further diagnosis and treatment.

The patient's abdomen was soft, with slight tenderness in the upper region. The laboratory results showed anemia (hemoglobin, $62 \mathrm{~g} / \mathrm{l}$; normal range, 120-160 g/l) and hypoproteinemia (albumine $23 \mathrm{~g} / \mathrm{l}$; normal range, 35-55 g/l), with total bilirubin (normal range, $0-21 \mu \mathrm{mol} / \mathrm{l}$ ), alanine aminotransferase 


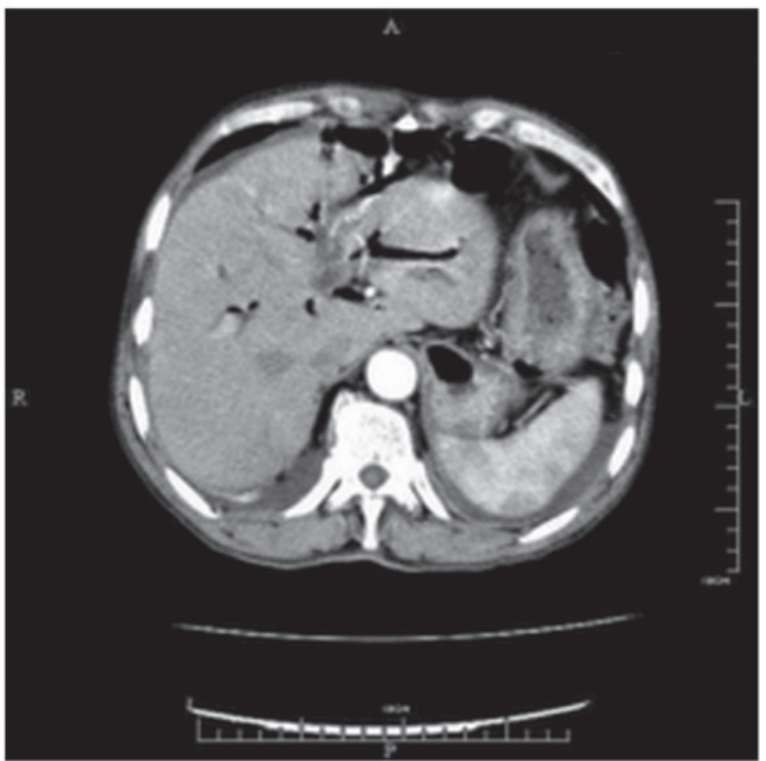

Figure 1. Abdominal computed tomography showing pneumobilia in the intrahepatic bile duct.
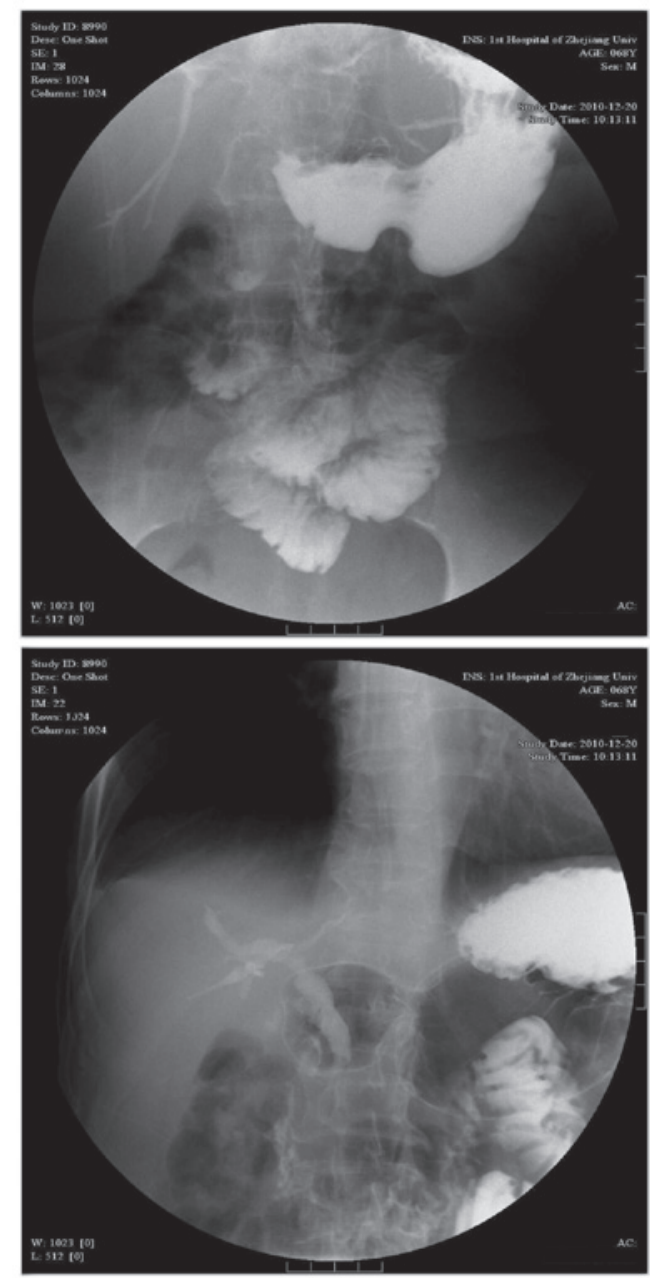

Figure 2. Gastroduodenography revealing a pyloric obstruction and contrast medium entering into the bile tract.

(normal range, 5-35 U/1) and aspartate transaminase (normal range, 8-40 U/l) levels that were all within the normal ranges. Anti-hemorrhagic (hemocoagulase atrox for injection,
4 units/day, intravenous injection ) and antibiotic treatments (Cefodizime Sodium, 2 g/day, intravenously guttae) were commenced. Abdominal ultrasound and magnetic resonance cholangiopancreatography showed multiple low-echo occupations and a stripe echo in the common bile duct, with dilatation of the intrahepatic and extrahepatic bile ducts. Abdominal computed tomography revealed stones in gallbladder and right liver with air in dilated intrahepatic and extrahepatic bile duct (Fig. 1). Suspecting that the bleeding was associated with the inflammation of the bile duct, endoscopic retrograde cholangiopancreatography (ERCP) was performed to find the position and the etiology of the bleeding. However, during the examination, the scope could not be passed through the obstructed section of deformed pylorus, and no significant abnormality of tract was found above the pylorus. Therefore, gastroduodenography was performed, which showed contrast medium entering into the bile tract (Fig. 2). Accordingly, upper GI bleeding and pyloric obstruction, with cholangitis secondary to a CDF was considered. Following anti-hemorrhagic and antibiotic treatments, the symptoms of fever and abdominal pain were relieved.

However, the patient received emergency surgery due to shock, which occurred 2 days later as a result of a massive hemorrhage of the GI tract. The hemoglobin level decreased to $40 \mathrm{~g} / \mathrm{l}$. During the surgery, the bleeding was located to a branch of the gastroduodenal artery, which was eroded by an ulcer ( $1.0 \mathrm{~cm}$ in diameter), in the pyloric canal. Furthermore, the scar of this ulcer was also believed to be responsible for the pyloric deformity. Additionally, another ulcer $(\sim 1.5 \mathrm{~cm}$ in diameter) was found around the area of the duodenal papilla, which was suspected to be the etiology for the disappearance of the papilla. No stones were found in the gallbladder or common bile duct during the procedure. So multiple surgeries, including a pancreatoduodenectomy, a pancreaticojejunostomy, a gastrojejunostomy and a choledochojejunostomy, were performed to fulfill the following three objectives: i) Control of the hemorrhage; ii) removal of the ulcers and the deformed pylorus; and iii) repair of the CDF. Finally, the post-operative pathology confirmed that the chronic ulcers in the pyloric canal and duodenum were benign. Following the surgery, the patient responded well and was discharged without any serious complications. The patient felt no discomfort associated with the former diseases and gastroscope found no ulcer recurrence during the following 3 years after the surgery.

\section{Discussion}

Bleeding is the most frequent of the multiple complications of peptic ulcers, occurring when an ulcer erodes a blood vessel. Therefore, it must be managed promptly and appropriately to prevent adverse outcomes. The guidelines for the management of patients with non-variceal upper GI bleeding recommend that endoscopy should generally be performed within $24 \mathrm{~h}$ in order to diagnose, assess and treat the bleeding lesion (3-5). Even when endoscopic therapy fails, certain useful information can be obtained for the subsequent transcatheter arterial embolization (TAE) or surgery. Where available, percutaneous or TAE has been investigated as an alternative to surgery in those individuals in whom endoscopic therapy has failed, particularly those patients who are high-risk candidates for surgery (4,6-9). 
A retrospective, single-center study (6) demonstrated that there were no significant differences between embolization therapy and surgery with regard to the rates of rebleeding, surgery or mortality, even though the patients in the embolization group were older with a higher prevalence of heart disease. In other studies, TAE was approved to associate with a high rate $(93 \%)$ of technical success, minimal complications (9\%), a decreased surgery rate and overall complications $(6,10,11)$. The indication for surgery is limited among cases where hemostasis cannot be performed due to the position of the ulcer or the type of bleeding. In the present patient, besides the GI bleeding, the pyloric obstruction and CDF also had to be taken into account. Therefore, endoscopic therapy and TAE were not suitable choices to solve all these problems.

Pyloric and duodenal obstructions always develop in patients with recurrent peptic ulcers as a result of acute inflammation, spasm, edema, or scarring and fibrosis. The patients suffer the associated symptoms of recurrent episodes of emesis, persistent abdominal distention or fullness after eating, early satiety and weight loss. Chronic peptic ulcer obstruction can be relieved by endoscopic pyloric balloon dilatation or surgery (vagotomy and pyloroplasty, antrectomy or gastroenterostomy) (12). However, former experience in our hospital showed that endoscopic balloon dilatation in pyloric obstruction does not achieve long-term remission, and the majority of patients require surgery.

CDF is a biliary abnormality that always occurs as a consequence of long-lasting and poorly-treated chronic duodenal ulcers $(13,14)$. Patients with this abnormality are believed to be prone to ascending biliary infection (15). The diagnosis of CDF is always incidentally made by radiography, with the signs of air in the biliary tree and barium reflux into the biliary tract following a barium meal (16). Demonstration of ostium discharging bile during ERCP is always regarded as the gold standard for the diagnosis of CDF. Possible treatments for this disease include conservative treatment with drugs, endoscopic sphincterotomy and surgical therapy. The choice is based on the etiology, the severity of the disease and the patient's general condition. It has been reported that recurrent bile tract infections and gallstone ileuses caused by CDF are considered as a definitive indication for surgical management (17). Presently, biliary enteric anastomosis is regarded as the primary method of treatment, with common bile duct exploration, T-tube drainage and endoscopic sphincterotomy also being effective $(15,18)$.

To the best of our knowledge, peptic ulcers accompanied with GI bleeding, pylorus obstruction and CDF have not previously been reported. In the present case, considering the coexisting complications of GI bleeding, pyloric obstruction and CDF, simple surgical intervention was insufficient. Therefore, a pancreatoduodenectomy was chosen for the purpose of simultaneously treating the bleeding artery, reconstructing the GI tract and resecting the CDF. The patient recovered well without any complications or remaining symptoms. Since peptic ulcers are extremely common, we hope this case will offer useful experience with regard to the diagnosis and treatment strategy for peptic ulcers that are accompanied by a group of complications.

\section{Acknowledgements}

The present study was supported by the Ministry of Health of Special Fund Industry (grant no. 201002015). The authors wish to thank all the doctors in the Division of Hepatobiliary and Pancreatic Surgery, Department of Surgery in The First Affiliated Hospital of Zhejiang University.

\section{References}

1. University of Michigan Health System. Peptic ulcer disease. www.cme.med.umich.edu/pdf/guideline/PUD05.pdf. Accessed May 4, 2007.

2. Malfertheiner P, Chan FK and McColl KE: Peptic ulcer disease. Lancet 374: 1449-1461, 2009.

3. Sung JJ, Chan FK, Chen M, Ching JY, Ho KY, Kachintorn U, Kim N, Lau JY, Menon J, Rani AA, et al; Asia-Pacific Working Group: Asia-Pacific Working Group consensus on non-variceal upper gastrointestinal bleeding. Gut 60: 1170-1177, 2011.

4. Barkun AN, Bardou M, Kuipers EJ, Sung J, Hunt RH, Martel M and Sinclair P; International Consensus Upper Gastrointestinal Bleeding Conference Group: International consensus recommendations on the management of patients with nonvariceal upper gastrointestinal bleeding. Ann Intern Med 152: 101-113, 2010.

5. Laursen SB, Jørgensen HS and Schaffalitzky de Muckadell OB; Danish Society of Gastroenterology and Hepatology: Management of bleeding gastroduodenal ulcers. Dan Med J 59: C4473, 2012.

6. Ripoll C, Bañares R, Beceiro I, Menchén P, Catalina MV Echenagusia A and Turegano F: Comparison of transcatheter arterial embolization and surgery for treatment of bleeding peptic ulcer after endoscopic treatment failure. J Vasc Interv Radiol 15: 447-450, 2004.

7. Larssen L, Moger T, Bjørnbeth BA, Lygren I and Kløw NE: Transcatheter arterial embolization in the management of bleeding duodenal ulcers: A 5.5-year retrospective study of treatment and outcome. Scand J Gastroenterol 43: 217-222, 2008.

8. Eriksson LG, Ljungdahl M, Sundbom M and Nyman R: Transcatheter arterial embolization versus surgery in the treatment of upper gastrointestinal bleeding after therapeutic endoscopy failure. J Vasc Interv Radiol 19: 1413-1418, 2008.

9. Venclauskas L, Bratlie SO,Zachrisson K, Maleckas A, Pundzius J and Jönson C: Is transcatheter arterial embolization a safer alternative than surgery when endoscopic therapy fails in bleeding duodenal ulcer? Scand J Gastroenterol 45: 299-304, 2010.

10. Loffroy R, Rao P, Ota S, De Lin M, Kwak BK and Geschwind JF: Embolization of acute nonvariceal upper gastrointestinal hemorrhage resistant to endoscopic treatment: Results and predictors of recurrent bleeding. Cardiovasc Intervent Radiol 33: 1088-1100, 2010.

11. Wong TC, Wong KT, Chiu PW, Teoh AY, Yu SC, Au KW and Lau JY: A comparison of angiographic embolization with surgery after failed endoscopic hemostasis to bleeding peptic ulcers. Gastrointest Endosc 73: 900-908, 2011.

12. Behrman SW: Management of complicated peptic ulcer disease. Arch Surg 140: 201-208, 2005.

13. Jorge A, Diaz M, Lorenzo J and Jorge O: Choledochoduodenal fistulas. Endoscopy 23: 76-78, 1991.

14. Colović R, Grubor N, Perisic M, Micev M, Latincić S and Colović N: Choledochoduodenal fistula of ulcer etiology. Srp Arh Celok Lek 138: 347-350, 2010 (In Serbian).

15. Choi D, Lim HK, Kim MJ, Kim SJ, Kim SH, Lee WJ, Lim JH, Paik SW, Yoo BC, Choi MS, et al: Liver abscess after percutaneous radiofrequency ablation for hepatocellular carcinomas: Frequency and risk factors. AJR Am J Roentgenol 184: 1860-1867, 2005

16. Zong KC, You HB, Gong JP and Tu B: Diagnosis and management of choledochoduodenal fistula. Am Surg 77: 348-350, 2011.

17. Vagefi PA, Ferguson CM and Hall JF: The occurrence of gallstone ileus. Arch Surg 143: 1118-1120, 2008.

18. Agarwal N, Sharma BC, Garg S, Kumar R and Sarin SK: Endoscopic management of postoperative bile leaks. Hepatobiliary Pancreat Dis Int 5: 273-277, 2006. 